\title{
Phonon-assisted nonradiative energy transfer from colloidal quantum dots to monocrystalline bulk silicon
}

\author{
Aydan Yeltik ${ }^{1}$, Burak Guzelturk ${ }^{1}$, Pedro Ludwig Hernandez Martinez ${ }^{1,2}$ and Hilmi Volkan Demir ${ }^{1,2}$ \\ ${ }^{1}$ Department of Electrical and Electronics Engineering, Department of Physics, UNAM - Institute of Materials Science and Nanotechnology, Bilkent \\ University, Ankara 06800 Turkey; ${ }^{2}$ School of Electrical and Electronic Engineering, School of Physical and Mathematical Sciences, Nanyang \\ Technological University, Nanyang Avenue, Singapore 639798, Singapore \\ *Tel:[+90](312) 2901021, e-mail: volkan@stanfordalumni.org
}

Silicon is one of the most dominant materials in photovoltaics. To increase optical absorption of silicon solar cells, colloidal quantum dots (QDs) have been proposed as a good sensitizer candidate owing to their favorably high absorption cross-section and tunable emission and absorption properties. To this end, QD sensitization of silicon has previously been studied by mostly facilitating radiative energy transfer (RET) [1,2]. Although RET based sensitization has achieved a considerable increase in conversion efficiencies in silicon photovoltaics, RET is fundamentally limited due to the effective coupling problem of emitted photons to silicon. Alternatively, nonradiative energy transfer (NRET), which relies on near field dipole-dipole coupling [3], has been shown to be feasible in sensitizer-silicon hybrid systems [4-8]. Although colloidal QDs as a sensitizer have been used to facilitate NRET into silicon, the detailed mechanisms of NRET to an indirect bandgap nonluminecent material, together with the role of phonon assistance and temperature activation, have not been fully understood to date. In this study, we propose a QD-silicon nanostructure hybrid platform to study the NRET dynamics as a function of temperature for distinct separation thicknesses between the donor QDs and the acceptor silicon plane. Here, we show NRET from colloidal QDs to bulk Si using phonon assisted absorption, developing its physical model to explain temperature-dependent lifetime dynamics of NRET in these QD-Si hybrids.

In this work, we used bulk monocrystalline p-type $\mathrm{Si}(100)$ substrate as the acceptor and core/shell CdSe/ZnS QDs as the donor. To match the absorption spectrum of silicon, QD peak emission wavelength was set to 580 $\mathrm{nm}$. Our Si substrates possessed approximately $1.65 \mathrm{~nm}$ thick native oxide on the top, as verified by the ellipsometry measurement. We deposited $\mathrm{Al}_{2} \mathrm{O}_{3}$ thin film on pre-cleaned $\mathrm{Si}$ substrates as a dielectric spacer using atomic layer deposition(ALD) technique. The film thicknesses of $\mathrm{Al}_{2} \mathrm{O}_{3}$ were carefully set to $0,1.0,2.0$ and 4.0 $\mathrm{nm}$ by ALD. The QDs were spin-coated over these $\mathrm{Al}_{2} \mathrm{O}_{3} / \mathrm{SiO}_{2} / \mathrm{Si}$ structures, resulting in formation of approximately 17 QD monolayers as measured by ellipsometry. Fluorescence decays of these QDs, which are furnished on the samples, were recorded by time resolved fluorescence spectroscopy with a closed cycle He cryostat at different temperatures varying between 22 and $290 \mathrm{~K}$. QD coated sapphire substrate was also utilized as the reference sample. As a result of the lifetime analysis using decaying exponential components, temperature-dependent exciton lifetimes of core/shell CdSe/ZnS QDs on $\mathrm{Al}_{2} \mathrm{O}_{3} / \mathrm{SiO}_{2} / \mathrm{Si}$ and sapphire substrates were obtained. Figure 1 presents (in red circles) amplitude averaged exciton lifetimes of CdSe/ZnS QDs deposited on $\mathrm{Al}_{2} \mathrm{O}_{3} / \mathrm{SiO}_{2} / \mathrm{Si}$ substrates for various $\mathrm{Al}_{2} \mathrm{O}_{3}$ thicknesses. Black squares are exciton lifetimes obtained for the QDs on the sapphire reference sample. These data were corrected for the difference in refractive index for sapphire and hypothetical Si having only a radiative decay lifetime in the way described by Novotny and Hecht [9]. It was observed that there is an increase in lifetimes with the decrease in temperature for all samples including the reference sample and the distinction between the lifetimes of the Si based samples and those of the reference sample becomes more pronounced at low temperatures.

To develop a theoretical understanding, we devised a physical model of NRET from the QD monolayers to bulk silicon, which uses phonon assistance in the absorption process [10]. Figure 2 illustrates the dipole-dipole Coulomb interaction between the donor QD and the acceptor Si. An absorbed photon in QD photogenerates an exciton in a higher excited state, relaxing rapidly to the first excited state through higher order processes. This exciton can subsequently be either recombined through a radiative or nonradiative means, or transferred to $\mathrm{Si}$ due to the Coulomb interaction between dipoles in the donor-acceptor pair. If the exciton in the QD is transferred to $\mathrm{Si}$, exciton lifetime of the QD decreases. Based on this phenomenon and using our energy transfer model we obtained lifetimes of the QDs on $\mathrm{Al}_{2} \mathrm{O}_{3} / \mathrm{SiO}_{2} / \mathrm{Si}$ substrates (considering contribution from each QD monolayer depending on its actual separation). In Figure 1, green down-triangles represent theoretical lifetimes extracted via our energy transfer model without temperature effects. As seen, there is a discrepancy between the experimental data and these theoretical lifetimes because the temperature effects are not included in the model. Therefore, we improved our model by introducing temperature effects on the parameters used. First, we included temperature dependency of the quantum yield for the QDs, which improved the theoretical prediction. However, this was not sufficient and there was still a discrepancy between the experimetal and theoretical lifetimes. This pointed out the need to account for $\mathrm{Si}$ absorption coefficient, which includes phonon term, as a function of temperature. As a result, shown as blue stars in Figure 1, we obtained the best matching results for the theoretical lifetimes of the QDs. This was attributed to the absorption properties of $\mathrm{Si}$, which is dictated by phonon assisted absorption due to its indirect band gap. All in all, our energy transfer model including phonon assistance in the 
absorption proved its effectiveness and reliability to assess temperature dependent time resolved fluorescence lifetimes.

In conclusion, we presented and analyzed the temperature-dependent fluorescence lifetimes in QD-silicon nanostructures to reveal dynamics of the NRET based on phonon assistance in the absorption process. To study the underpinning physics of this NRET, we used QD-silicon nanostructure platforms of different separation thicknesses between the donor QDs and the acceptor silicon. As a theoretical approach, we suggested a new physical model including temperature effects based on phonon-assisted absorption and concluded that it proves to be succesful in the temperature dependence analysis of the time-resolved fluorescence lifetimes. Therefore, this work reveals that phonons play a critical role in NRET to Si acceptor as an indirect bandgap semiconductor as they do in the optical absorption.
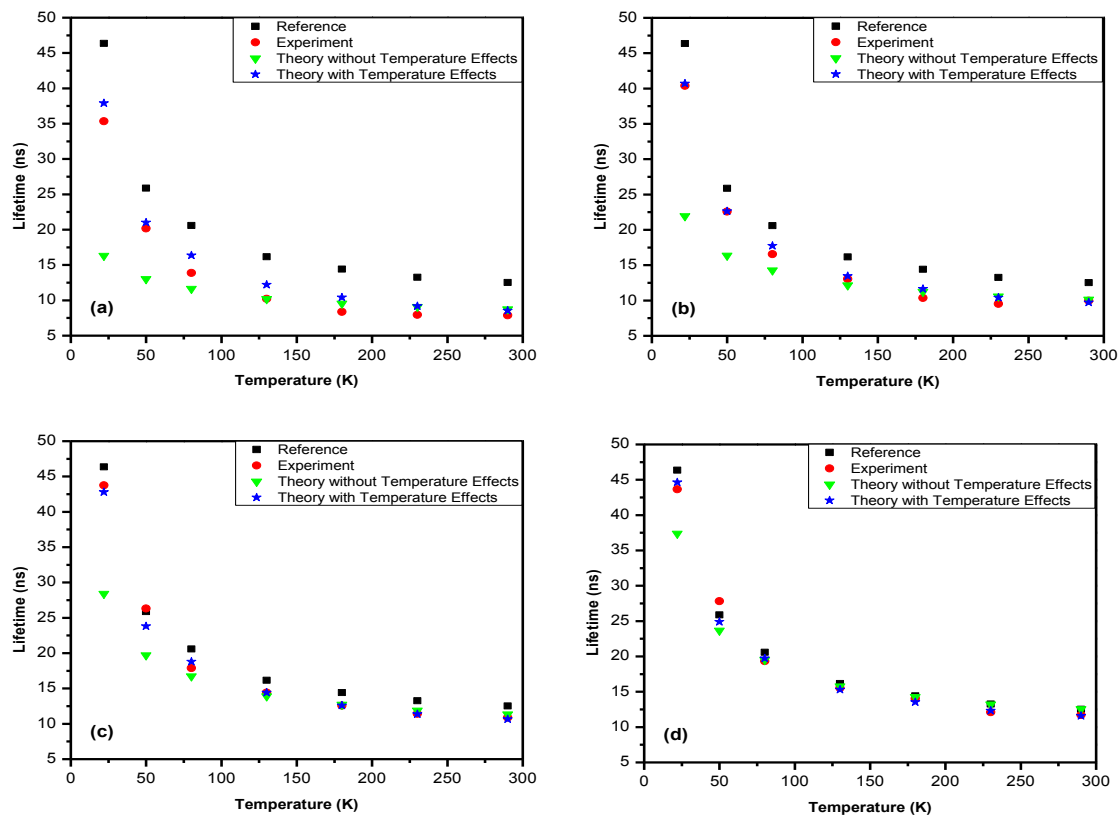

Figure 1. Temperature dependence of exciton lifetimes of $\mathrm{CdSe} / \mathrm{ZnS}$ QDs integrated on top of (a) $0 \mathrm{~nm}$ thick (none) $\mathrm{Al}_{2} \mathrm{O}_{3}$, (b) $1.0 \mathrm{~nm}$ thick $\mathrm{Al}_{2} \mathrm{O}_{3}$, (c) 2.0nm thick $\mathrm{Al}_{2} \mathrm{O}_{3}$ and (d) 4.0nm thick $\mathrm{Al}_{2} \mathrm{O}_{3}$ deposited on $\mathrm{SiO}_{2} / \mathrm{Si}$ (red circles). Black squares are the experimental exciton lifetimes for the QDs on sapphire as the reference sample. Green down-triangles are the theoretical lifetimes of the QDs using the energy transfer model without temperature effects. Blue stars are the theoretical lifetimes of the QDs using the energy transfer model with temperature effects based on phonon-assisted absorption.

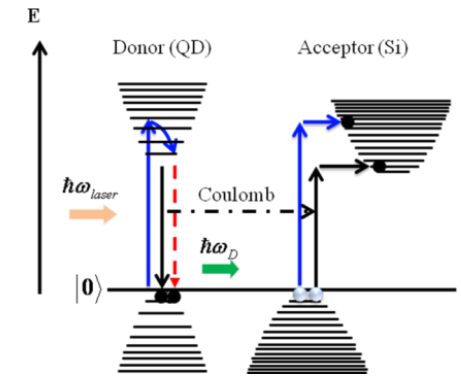

Figure 2. Energy diagram for the illustration of exciton migration from the donor QD to the acceptor Si due to the Coulomb interaction between the donor-acceptor pair. The phonon assistance is shown as the lateral arrows to make up for the momentum mismatch in Si.

Acknowledgements: This work is supported in part by EU-FP7 Nanophotonics4Energy NoE, and TUBITAK EEEAG, 109E002, 109E004, 110E010, and 110E217, and in part by NRF-CRP-6-2010-02 and NRF-RF-2009-09. H.V.D. acknowledges support from ESF-EURYI and TUBA-GEBIP; and A.Y. from TUBITAK BIDEB.

References

[1] E. Mutlugun et al., Opt. Express 16, 3537, (2008).

[2] B. Guzelturket al., Appl. Phys. Lett., 97(9), 093111, (2010).

[3] T. Förster, Ann. Phys. 437, 55, (1948).

[4] M. Stavola et al., Phys. Rev. B 31, 2277, (1985).

[5] A. P. Alivisatos et al., J. Chem. Phys. 86, 6540, (1987).

[6] S. Lu and A. Madhukar, Nano Lett., 7, 3443-3451, (2007).

[7] S. Lu et al., Nano Lett., 9, 4548, (2009).

[8] H. M. Nguyen et al., Appl. Phys. Lett., 98, 161904, (2011).

[9] L. Novotny and B. Hecht, Cambridge University Press, (2006).

[10] A. Yeltik et al., (2012) (submitted). 